\title{
Strain differences in organ weights of behaviorally defined rats
}

\author{
GORDON M. HARRINGTON and LOUIS R. HELLWIG \\ University of Northern Iowa, Cedar Falls, Iowa 50613
}

\begin{abstract}
Adrenal, brain, pituitary, spleen, thymus, and thyroid weights were obtained for 577 rats from 12 inbred strains: ACI, A990, A35322, F344, INR, IR, MNR/Har, MNRA, MR/Har, TS1, TS3, WAG. The results provide parametric data for methodological use and add to the standardization of these strains as behaviorally defined lines.
\end{abstract}

The rat was the first animal to be domesticated for scientific purposes and was first used for study of the adrenals (Philipeaux, 1856). Adrenal and other endocrine weights have been an important variable in behavioral studies since Watson (1907) observed adrenal differences between wild and laboratory rats. Parallel brain-weight differences were observed shortly thereafter by H. H. Donaldson and Hatai (1911). Subsequently, J. C. Donaldson (1923) found evidence suggesting that organ-weight variations between wild and tame rats were attributable both to genetic factors and to weaning stress. Experimental study of relationships of endocrine and brain weights to defecation and to maze learning was well established before World War II (Anderson \& Anderson, 1938; Silverman, Shapiro, \& Heron, 1940; Yeakel \& Rhoades, 1941). The present study was carried out to provide parametric data on organ weights in a number of well-specified lines of rats.

The last decade has seen significant conceptual and methodological advances in the life sciences in definition of animal subjects (International Committee on Laboratory Animals, 1971). These developments seem to have had least impact on behavioral experimentation. Although the rat has outranked all other infrahumans as the animal of preference for behavioral studies, there has been a dearth of behavioral data enabling either informed choice or precise specification of subjects. This study of organ weights is one of a number of studies (Harrington, 1971a, 1971b, 1979a, 1979b, 1979c, 1979d, 1979e, 1979f, 1979g, 1979h, 1979i; Harrington \& Hellwig, 1979) cataloging a variety of characteristics for behavioral standardization of the 12 strains of rats that have had the highest citation frequency in the behavioral literature.

\section{METHOD}

Subjects

Subjects were 577 rats, aged 132-385 (mean $=216, \mathrm{SD}=58)$ days, with a minimum of 20 animals of each sex from each of
12 strains bred and maintained in the writers' laboratory. All animals were maintained at $2.5^{\circ} \mathrm{C} \pm 1.1^{\circ} \mathrm{C}$ and $40 \% \pm 5 \%$ relative humidity. Breeders and pups were maintained on a natural light cycle. Pups were handled for 1 -min periods on alternate days from 14 to 45 days of age and transferred to 24-h light conditions at 45 days. Strains were ACI/Har, A990/ Har, A35322/Har, F344/DuHar, INR, IR, MNR/Har, MNRA (formerly MNR-a/Har), MR/Har, TS1, TS3, and WAG/Har. All strains are identified in the fourth international listing of inbred strains (Festing \& Staats, 1973) and are described in more detail elsewhere (Harrington, 1968).

\section{Procedure}

Chloroform was administered to animals individually by inhalation in an enclosed cage until cessation of respiration. The animal was then weighed. Immediate necropsy followed. The left and right adrenals, the pituitary, the spleen, the thymus, the thyroid including parathyroid III (Ruch \& Patton, 1965), and the brain excluding olfactory bulbs were removed and weighed without delay.

\section{RESULTS AND DISCUSSION}

Organ weights were divided by animal weight. The resulting data distributions are shown in Table 1, expressed in milligrams per killograms of body weight.

The differences in adrenal weights for the MNR, MNRA, and MR lines are consistent with data previously reported, but the thyroid differences are not (Feuer \& Broadhurst, 1961a, 1961b, 1961c). With respect to the thyroid data, it should be particularly noted that wet weights are reported here and also by Feuer and Broadhurst. Broadhurst (1960) earlier reported dryweight thyroid differences in the same direction found here with wet weights and thus in the opposite direction to his wet-weight data. This suggests a need for closer exploration of the relationship between wet-weight and dry-weight measures.

The brain-weight differences between TS1 and TS3 are consistent with those obtained on the ancestral animals from which these strains were subsequently derived (Bennet, Rosenzweig, Krech, Karlsson, Dye, \& Ohlander, 1958). 
Table 1

Organ Weights of 12 Inbred Strains of Laboratory Rats

\begin{tabular}{|c|c|c|c|c|c|c|c|c|c|c|c|c|c|c|}
\hline \multirow[b]{2}{*}{ Strain } & \multicolumn{2}{|c|}{ Left Adrenal } & \multicolumn{2}{|c|}{ Right Adrenal } & \multicolumn{2}{|c|}{ Pituitary } & \multicolumn{2}{|c|}{ Spleen } & \multicolumn{2}{|c|}{ Thymus } & \multicolumn{2}{|c|}{ Thyroid } & \multicolumn{2}{|c|}{ Brain } \\
\hline & Mean & SD & Mean & SD & Mean & SD & Mean & SD & Mean & SD & Mean & SD & Mean & SD \\
\hline $\begin{array}{l}\text { ACI/Har } \\
\text { Males } \\
\text { Females }\end{array}$ & $\begin{array}{r}57.4 \\
110.0\end{array}$ & $\begin{array}{l}9.3 \\
9.0\end{array}$ & $\begin{array}{l}50.3 \\
97.0\end{array}$ & $\begin{array}{r}5.8 \\
13.6\end{array}$ & $\begin{array}{l}33.2 \\
58.1\end{array}$ & $\begin{array}{l}3.5 \\
9.3\end{array}$ & $\begin{array}{l}1541 \\
1819\end{array}$ & $\begin{array}{r}85 \\
139\end{array}$ & $\begin{array}{l}537 \\
976\end{array}$ & $\begin{array}{r}81 \\
153\end{array}$ & $\begin{array}{l}61.4 \\
82.5\end{array}$ & $\begin{array}{l}12.9 \\
15.0\end{array}$ & $\begin{array}{l}7193 \\
9944\end{array}$ & $\begin{array}{l}456 \\
487\end{array}$ \\
\hline $\begin{array}{l}\text { A990/Har } \\
\text { Males } \\
\text { Females }\end{array}$ & $\begin{array}{r}47.3 \\
107.7\end{array}$ & $\begin{array}{r}9.4 \\
20.6\end{array}$ & $\begin{array}{l}45.7 \\
97.2\end{array}$ & $\begin{array}{r}7.2 \\
16.8\end{array}$ & $\begin{array}{l}25.9 \\
55.6\end{array}$ & $\begin{array}{r}5.6 \\
12.4\end{array}$ & $\begin{array}{l}1721 \\
2242\end{array}$ & $\begin{array}{l}133 \\
213\end{array}$ & $\begin{array}{l}430 \\
779\end{array}$ & $\begin{array}{l}126 \\
294\end{array}$ & $\begin{array}{l}67.0 \\
90.3\end{array}$ & $\begin{array}{l}19.0 \\
23.2\end{array}$ & $\begin{array}{l}5303 \\
8362\end{array}$ & $\begin{array}{l}506 \\
576\end{array}$ \\
\hline $\begin{array}{l}\text { A35322/Ha } \\
\text { Males } \\
\text { Females }\end{array}$ & $\begin{array}{l}47.9 \\
90.3\end{array}$ & $\begin{array}{l}6.6 \\
9.2\end{array}$ & $\begin{array}{l}42.2 \\
73.6\end{array}$ & $\begin{array}{r}5.3 \\
10.5\end{array}$ & $\begin{array}{l}31.0 \\
48.8\end{array}$ & $\begin{array}{l}3.2 \\
6.9\end{array}$ & $\begin{array}{l}2366 \\
2705\end{array}$ & $\begin{array}{l}178 \\
181\end{array}$ & $\begin{array}{l}514 \\
725\end{array}$ & $\begin{array}{l}199 \\
192\end{array}$ & $\begin{array}{l}58.2 \\
84.2\end{array}$ & $\begin{array}{l}16.5 \\
15.9\end{array}$ & $\begin{array}{l}6682 \\
9473\end{array}$ & $\begin{array}{l}757 \\
534\end{array}$ \\
\hline $\begin{array}{l}\text { F344/DuHa } \\
\text { Males } \\
\text { Females }\end{array}$ & $\begin{array}{l}41.0 \\
79.2\end{array}$ & $\begin{array}{r}8.2 \\
17.3\end{array}$ & $\begin{array}{l}39.3 \\
75.4\end{array}$ & $\begin{array}{r}9.4 \\
13.3\end{array}$ & $\begin{array}{l}31.7 \\
60.4\end{array}$ & $\begin{array}{r}5.8 \\
13.4\end{array}$ & $\begin{array}{l}1585 \\
2099\end{array}$ & $\begin{array}{l}102 \\
140\end{array}$ & $\begin{array}{l}451 \\
743\end{array}$ & $\begin{array}{l}147 \\
210\end{array}$ & $\begin{array}{l}63.7 \\
84.0\end{array}$ & $\begin{array}{l}15.2 \\
22.8\end{array}$ & $\begin{array}{l}5899 \\
9034\end{array}$ & $\begin{array}{l}753 \\
547\end{array}$ \\
\hline $\begin{array}{l}\text { INR } \\
\text { Males } \\
\text { Females }\end{array}$ & $\begin{array}{l}38.3 \\
79.5\end{array}$ & $\begin{array}{r}7.1 \\
12.6\end{array}$ & $\begin{array}{l}34.4 \\
72.5\end{array}$ & $\begin{array}{r}6.2 \\
11.5\end{array}$ & $\begin{array}{l}26.2 \\
51.8\end{array}$ & $\begin{array}{r}5.8 \\
11.6\end{array}$ & $\begin{array}{l}1816 \\
2056\end{array}$ & $\begin{array}{l}238 \\
161\end{array}$ & $\begin{array}{l}681 \\
986\end{array}$ & $\begin{array}{l}209 \\
206\end{array}$ & $\begin{array}{l}66.0 \\
82.5\end{array}$ & $\begin{array}{l}12.5 \\
11.6\end{array}$ & $\begin{array}{l}5258 \\
8076\end{array}$ & $\begin{array}{l}847 \\
664\end{array}$ \\
\hline $\begin{array}{l}\text { IR } \\
\text { Males } \\
\text { Females }\end{array}$ & $\begin{array}{l}36.0 \\
71.6\end{array}$ & $\begin{array}{r}7.6 \\
15.4\end{array}$ & $\begin{array}{l}29.9 \\
64.3\end{array}$ & $\begin{array}{r}7.0 \\
11.8\end{array}$ & $\begin{array}{l}34.3 \\
57.8\end{array}$ & $\begin{array}{l}3.1 \\
5.6\end{array}$ & $\begin{array}{l}1247 \\
1544\end{array}$ & $\begin{array}{l}124 \\
123\end{array}$ & $\begin{array}{r}610 \\
1027\end{array}$ & $\begin{array}{l}222 \\
252\end{array}$ & $\begin{array}{l}53.4 \\
68.9\end{array}$ & $\begin{array}{l}10.4 \\
17.4\end{array}$ & $\begin{array}{l}6432 \\
9002\end{array}$ & $\begin{array}{l}613 \\
606\end{array}$ \\
\hline $\begin{array}{l}\text { MNR/Har } \\
\text { Males } \\
\text { Females }\end{array}$ & $\begin{array}{r}46.7 \\
105.2\end{array}$ & $\begin{array}{r}7.3 \\
13.0\end{array}$ & $\begin{array}{l}42.4 \\
96.3\end{array}$ & $\begin{array}{l}10.0 \\
17.3\end{array}$ & $\begin{array}{l}30.7 \\
65.3\end{array}$ & $\begin{array}{l}3.3 \\
8.1\end{array}$ & $\begin{array}{l}1539 \\
1883\end{array}$ & $\begin{array}{l}125 \\
113\end{array}$ & $\begin{array}{r}630 \\
1109\end{array}$ & $\begin{array}{l}240 \\
264\end{array}$ & $\begin{array}{l}59.1 \\
77.4\end{array}$ & $\begin{array}{l}14.2 \\
21.5\end{array}$ & $\begin{array}{r}6744 \\
10230\end{array}$ & $\begin{array}{l}651 \\
583\end{array}$ \\
\hline $\begin{array}{l}\text { MNRA } \\
\text { Males } \\
\text { Females }\end{array}$ & $\begin{array}{r}45.1 \\
104.0\end{array}$ & $\begin{array}{r}8.9 \\
22.6\end{array}$ & $\begin{array}{l}41.5 \\
94.2\end{array}$ & $\begin{array}{r}6.7 \\
20.7\end{array}$ & $\begin{array}{l}29.4 \\
53.0\end{array}$ & $\begin{array}{l}6.2 \\
9.0\end{array}$ & $\begin{array}{l}1781 \\
2203\end{array}$ & $\begin{array}{l}243 \\
226\end{array}$ & $\begin{array}{l}512 \\
852\end{array}$ & $\begin{array}{l}127 \\
143\end{array}$ & $\begin{array}{l}66.2 \\
86.4\end{array}$ & $\begin{array}{l}13.5 \\
19.5\end{array}$ & $\begin{array}{r}6975 \\
10953\end{array}$ & $\begin{array}{l}536 \\
551\end{array}$ \\
\hline $\begin{array}{l}\text { MR/Har } \\
\text { Males } \\
\text { Females }\end{array}$ & $\begin{array}{r}49.4 \\
104.7\end{array}$ & $\begin{array}{r}8.1 \\
25.0\end{array}$ & $\begin{array}{l}45.0 \\
97.8\end{array}$ & $\begin{array}{r}9.4 \\
13.2\end{array}$ & $\begin{array}{l}31.4 \\
48.8\end{array}$ & $\begin{array}{r}4.6 \\
10.4\end{array}$ & $\begin{array}{l}2214 \\
2701\end{array}$ & $\begin{array}{l}187 \\
296\end{array}$ & $\begin{array}{r}720 \\
1094\end{array}$ & $\begin{array}{l}145 \\
240\end{array}$ & $\begin{array}{l}48.0 \\
76.0\end{array}$ & $\begin{array}{l}15.0 \\
21.7\end{array}$ & $\begin{array}{l}5986 \\
9603\end{array}$ & $\begin{array}{l}457 \\
632\end{array}$ \\
\hline $\begin{array}{l}\text { TS1 } \\
\text { Males } \\
\text { Females }\end{array}$ & $\begin{array}{l}44.6 \\
91.7\end{array}$ & $\begin{array}{l}11.0 \\
16.3\end{array}$ & $\begin{array}{l}38.5 \\
81.6\end{array}$ & $\begin{array}{r}7.9 \\
12.5\end{array}$ & $\begin{array}{l}34.4 \\
54.3\end{array}$ & $\begin{array}{l}5.0 \\
9.5\end{array}$ & $\begin{array}{l}1628 \\
2109\end{array}$ & $\begin{array}{l}122 \\
160\end{array}$ & $\begin{array}{l}506 \\
662\end{array}$ & $\begin{array}{l}131 \\
152\end{array}$ & $\begin{array}{l}70.4 \\
93.8\end{array}$ & $\begin{array}{l}12.3 \\
13.0\end{array}$ & $\begin{array}{l}4775 \\
7596\end{array}$ & $\begin{array}{l}515 \\
533\end{array}$ \\
\hline $\begin{array}{l}\text { TS3 } \\
\text { Males } \\
\text { Females }\end{array}$ & $\begin{array}{l}42.5 \\
75.0\end{array}$ & $\begin{array}{r}6.7 \\
14.5\end{array}$ & $\begin{array}{l}37.0 \\
65.2\end{array}$ & $\begin{array}{r}5.4 \\
12.8\end{array}$ & $\begin{array}{l}32.1 \\
49.0\end{array}$ & $\begin{array}{l}6.2 \\
7.5\end{array}$ & $\begin{array}{l}1864 \\
2358\end{array}$ & $\begin{array}{l}245 \\
145\end{array}$ & $\begin{array}{l}366 \\
623\end{array}$ & $\begin{array}{l}130 \\
192\end{array}$ & $\begin{array}{l}53.4 \\
79.0\end{array}$ & $\begin{array}{l}10.0 \\
17.6\end{array}$ & $\begin{array}{l}5462 \\
7809\end{array}$ & $\begin{array}{l}608 \\
621\end{array}$ \\
\hline $\begin{array}{l}\text { WAG/Har } \\
\text { Males } \\
\text { Females }\end{array}$ & $\begin{array}{l}40.1 \\
62.3\end{array}$ & $\begin{array}{l}8.1 \\
9.9\end{array}$ & $\begin{array}{l}33.8 \\
60.1\end{array}$ & $\begin{array}{r}7.2 \\
13.4\end{array}$ & $\begin{array}{l}31.5 \\
53.7\end{array}$ & $\begin{array}{l}6.0 \\
8.9\end{array}$ & $\begin{array}{l}1344 \\
1644\end{array}$ & $\begin{array}{l}183 \\
102\end{array}$ & $\begin{array}{r}674 \\
1158\end{array}$ & $\begin{array}{l}269 \\
231\end{array}$ & $\begin{array}{l}57.4 \\
79.0\end{array}$ & $\begin{array}{l}18.2 \\
13.0\end{array}$ & $\begin{array}{l}5803 \\
9232\end{array}$ & $\begin{array}{r}1029 \\
663\end{array}$ \\
\hline
\end{tabular}

Note $-N \geqslant 20$ for each sex within each strain.

\section{REFERENCES}

Anderson, E. E., \& Anderson, S. F. The relation between the weight of the end ocrine glands and measures of sexual, emotional and exploratory behavior in the male albino rat. Journal of Comparative Psychology, 1938, 26, 459-474.

Bennett, E. L., RosenzWeig, M. R., Krech, D., Karlsson, H., DYE, N., \& OHLANDER, A. Individual strain and age differences in cholinesterase activity of the rat brain. Journal of Neurochemistry, 1958, 3, 144-152.

BroAdHURST, P. L. Experiments in psychogenetics: Applications of biometrical genetics to the inheritance of behavior. In H. J. Eysenck (Ed.), Experiments in personality: Psychogenetics and psychopharmacology (Vol. 1). London: Routledge \& Kegan Paul, 1960. Pp. 1-102.

Donaldson, H. H., \& Hatai, S. A comparison of the Norway rat with the albino rat in respect to body length, brain weight, spinal cord weight and the percentage of water in both the brain and the spinal cord. Journal of Comparative Neurology, 1911, 21, 417-458.

Donaldson, J. C. Note on the weight of the adrenals in crosses between albino and the wild Norway rat. Proceedings of the Society for Experimental Biology, 1923, 21, 157-160.
Festing, M., \& StaAts, J. Standardized nomenclature for inbred strains of rats. Transplantation, 1973, 16, 221-245.

Feuer, G., \& Broadhurst, P. L. Thyroid function in rats selectively bred for emotional elimination. I. Differences in thyroid hormone. Journal of Endocrinology, 1962, 24, 127-136. (a)

Feuer, G., \& Broadhurst, P. L. Thyroid function in rats selectively bred for emotional elimination. II. Differences in thyroid activity. Journal of Endocrinology, 1962, 24, 253-262. (b)

Feuer, G., \& Brondhurst, P. L. Thyroid function in rats selectively bred for emotional elimination. III. Behavioral differences. Journal of Endocrinology, 1962, 24, 385-396. (c)

HARRINGTON, G. M. Genetic-environmental interaction in "intelligence." I: Biometric genetic analysis of maze performance of Rattus Norvegicus. Developmental Psychobiology, 1968, 1, 211-218.

HARRINGTON, G. M. Strain differences among rats initiating exploration of differing environments. Psychonomic Science, 1971, 23, 348-349. (a)

HARrington, G. M. Strain differences in rotating wheel activity of the rat. Psychonomic Science, 1971, 23, 363-364. (b)

HARRINGton, G. M. Strain differences in open field behavior of the rat. Psychonomic Science, 1972, 27, 51-53.

HARRINGTON, G. M. Strain differences in activity of the rat in a 
shuttle stabilimeter. Bulletin of the Psychonomic Society, 1979, 13, 149-150. (a)

HARRINGTON, G. M. Strain differences in activity of the rat using a home cage stabilimeter. Bulletin of the Psychonomic Society, 1979, 13, 151-152. (b)

HARRINGTON, G. M. Strain differences in free operant leverpress levels in the rat. Bulletin of the Psychonomic Society, 1979, 13, 153-154. (c)

HARRINGTON, G. M. Strain differences in light-contingent barpress behavior of the rat. Bulletin of the Psychonomic Society, 1979, 13, 155-156. (d)

HARrington, G. M. Strain differences in open-field behavior of the rat. II. Bulletin of the Psychonomic Society, 1979, 13, 85-86. (e)

HARRINGTON, G. M. Strain differences in passive avoidance conditioning in the rat. Bulletin of the Psychonomic Society, 1979, 13, 157-158. (f)

HARRINGTON, G. M. Strain differences in runway learning in the rat. Bulletin of the Psychonomic Society, 1979, 13, 159-160. (g)

HARRINGTON, G. M. Strain differences in shuttle avoidance conditioning in the rat. Bulletin of the Psychonomic Society, 1979, 13, 161-162. (h)

HARRINGTON, G. M. Strain differences in simple operant barpress acquisition to an auditory stimulus by rats. Bulletin of the
Psychonomic Society, 1979, 13, 163-164. (i)

HarRington, G. M., \& Hellwig, L. R. Strain differences in basal metabolism of behaviorally defined rats. Bulletin of the Psychonomic Society, 1979, 13, 165-166.

International Committee on Laboratory Animals. Defining the laboratory animal. Washington, D.C: National Academy of Sciences, 1971.

Philipeaux, J. M. Note sur l'exterpation des capsules surrenales chez les rats albinos (Mus rattus). Compt-rendu Academie Sciences, Paris, 1856, 43, 904-906.

Ruch, T. C., \& Patron, H. D. Physiology and biophysics (19th ed.). Philadelphia: Saunders, 1965.

Silverman, W., Shapiro, F., \& Heron, W. T. Brain weight and maze learning in rats. Journal of Comparative Psychology, 1940, 30, 279-282.

Watson, C. A note on the adrenal gland in the rat. Journal of Physiology, 1907, 35, 230-232.

YEAKel, E. H., \& RhoADES, R. P. A comparison of the body and endocrine gland (adrenal, thyroid and pituitary) weights of emotional and non-emotional rats. Endocrinology, 1941, 28, 337-340.

(Received for publication January 3, 1979.) 